\title{
Endovascular treatment for acute ischaemic stroke in routine clinical practice: prospective, observational cohort study (MR CLEAN Registry)
}

\author{
Ivo G H Jansen, Maxim J H L Mulder, Robert-Jan B Goldhoorn, et al for the MR CLEAN Registry \\ investigators
}

Correspondence to:

Ivo G H Jansen

i.g.jansen@amc.nl

Additional material is published online only. To view please visit the journal online.

Cite this as: BMJ 2018;360:k949 http://dx.doi.org/10.1136/bmj.k949

Accepted: 21 February 2018

\section{ABSTRACT}

OBJECTIVE

To determine outcomes and safety of endovascular treatment for acute ischaemic stroke, due to proximal intracranial vessel occlusion in the anterior circulation, in routine clinical practice.

\section{DESIGN}

Ongoing, prospective, observational cohort study.

SETTING

16 centres that perform endovascular treatment in the Netherlands.

\section{PARTICIPANTS}

1488 patients included in the Multicentre Randomised Controlled Trial of Endovascular Treatment for Acute Ischaemic Stroke in the Netherlands (MR CLEAN) Registry who had received endovascular treatment, including stent retriever thrombectomy, aspiration, and all alternative methods for acute ischaemic stroke within 6.5 hours from onset of symptoms between March 2014 and June 2016.

\section{MAIN OUTCOME MEASURES}

The primary outcome was the modified Rankin Scale (mRS) score, ranging from 0 (no symptoms) to 6 (death) at 90 days after the onset of symptoms. Secondary outcomes were excellent functional outcome (mRS score $0-1$ ), good functional outcome (mRS score 0-2), and favourable functional outcome (mRS score 0-3) at 90 days; score on the extended thrombolysis in cerebral infarction scale at the end of the intervention procedure; National Institutes of Health Stroke Scale score 24-48 hours after

\section{WHAT IS ALREADY KNOWN ON THIS TOPIC}

Several randomised trials and meta-analyses have shown efficacy and safety of endovascular treatment for acute ischaemic stroke due to proximal intracranial vessel occlusion in the anterior circulation, within six hours from symptom onset Whether outcomes and safety in routine clinical practice are comparable to previous randomised clinical trials of endovascular treatment for acute ischaemic stroke is unknown

Current information about outcomes and safety of endovascular treatment is derived from patients treated in the setting of a randomised controlled trial

\section{WHAT THIS STUDY ADDS}

The results of the MR CLEAN Registry show that in routine clinical practice, endovascular treatment for patients with acute ischaemic stroke due to proximal intracranial vessel occlusion in the anterior circulation is at least as effective and safe as in the setting of a randomised controlled trial

The findings confirm that endovascular treatment should be standard of care for patients with acute ischaemic stroke due to proximal intracranial vessel occlusion in the anterior circulation intervention; and complications that occurred during intervention, hospital admission, or three months' follow up period. Outcomes and safety variables in the MR CLEAN Registry were compared with the MR CLEAN trial intervention and control arms.

\section{RESULTS}

A statistically significant shift was observed towards better functional outcome in patients in the MR CLEAN Registry compared with the MR CLEAN trial intervention arm (adjusted common odds ratio 1.30, $95 \%$ confidence interval 1.02 to 1.67 ) and the MR CLEAN trial control arm $(1.85,1.46$ to 2.34$)$. The reperfusion rate, with successful reperfusion defined as a score of $2 \mathrm{~B}-3$ on the extended thrombolysis in cerebral infarction score, was $58.7 \%$, the same as for patients in the MR CLEAN trial. Duration from onset of stroke to start of endovascular treatment and from onset of stroke to successful reperfusion or last contrast bolus was one hour shorter for patients in the MR CLEAN Registry. Symptomatic intracranial haemorrhage occurred in $5.8 \%$ of patients in the MR CLEAN Registry compared with $7.7 \%$ in the MR CLEAN trial intervention arm and $6.4 \%$ in the MR CLEAN trial control arm.

\section{CONCLUSION}

In routine clinical practice, endovascular treatment for patients with acute ischaemic stroke is at least as effective and safe as in the setting of a randomised controlled trial.

\section{Introduction}

Endovascular treatment for acute ischaemic stroke, due to proximal intracranial vessel occlusion in the anterior circulation, has been shown to be effective and safe in several randomised controlled trials and subsequent meta-analyses. $^{1-9}$ In the Multicentre Randomised Controlled Trial of Endovascular Treatment for Acute Ischaemic Stroke in the Netherlands (MR CLEAN) good functional outcome was clearly more often reached with endovascular treatment (33\%) than without $(19 \%) .^{2}$ In the pooled analysis of individual patient data of the first five randomised controlled trials with positive results, the number needed to treat to reduce disability was $2.6 .^{9}$ However, these results were achieved within the confines of randomised controlled trials with strict inclusion and exclusion criteria. The MR CLEAN trial had the most broadly defined inclusion criteria of these randomised controlled trials, bearing in mind that doctor judgment most likely played a role in patient enrolment. Still, external validity of results acquired in randomised trials is always a point 
of concern, as optimistic results might arise from selective inclusion, strict monitoring of procedures, and early termination. ${ }^{1011}$

Currently, endovascular treatment has become the standard of care in many countries. In the Netherlands, the implementation of endovascular treatment as standard treatment for patients with acute ischaemic stroke due to proximal intracranial vessel occlusion is monitored in the MR CLEAN Registry. We determined outcomes and safety of endovascular treatment in routine clinical practice by comparing outcomes (clinical, imaging, and time characteristics) of patients in the MR CLEAN Registry with those in the MR CLEAN trial.

\section{Methods \\ Design}

The MR CLEAN Registry is an ongoing, prospective, observational study in all centres that perform endovascular treatment in the Netherlands. Until 1 January 2017, endovascular treatment for acute ischaemic stroke was only reimbursed in the Netherlands if patients were treated in centres that participated in the MR CLEAN trial or the MR CLEAN Registry. All centres that participated in the MR CLEAN trial registered patients who received endovascular treatment, and we invited new intervention centres in the Netherlands performing endovascular treatment to participate.

\section{Patient enrolment}

Enrolment in the MR CLEAN Registry started directly after the final randomisation in the MR CLEAN trial on 16 March 2014. From 16 March 2014 to 31 December 2014 this was done retrospectively. From January 2015 onwards, enrolment was prospective. Sixteen centres participated in the MR CLEAN trial and are considered "MR CLEAN centres." Two non-MR CLEAN centres started performing endovascular treatment later on and added patients to the MR CLEAN Registry, but these patients are not included in this analysis. The study data for patients undergoing endovascular treatment up to 15 June 2016 in the 16 MR CLEAN centres were completed and analysed and are reported here.

All patients undergoing endovascular treatment (defined as entry into the angiography suite and receiving arterial puncture) for acute ischaemic stroke in the anterior and posterior circulation have been registered in the MR CLEAN Registry. To adequately compare results with the MR CLEAN trial, in the current analysis we included those patients who adhered to the following criteria: arterial puncture within 6.5 hours of symptom onset, age 18 years and older, treatment in a centre that participated in the MR CLEAN trial, and proximal intracranial vessel occlusion in the anterior circulation (internal carotid artery (ICA), internal carotid artery terminus (ICA-T), middle (M1/M2) cerebral artery, or anterior (A1/A2) cerebral artery), shown by computed tomography angiography, magnetic resonance angiography, or digital subtraction angiography. No upper age limit, minimum Alberta Stroke Program Early Computed
Tomography Score (ASPECTS), or collateral grade were imposed on treating doctors, nor was an extracranial occlusion by atherosclerosis or dissection an exclusion criterion.

\section{Outcomes}

The primary outcome was the modified Rankin Scale (mRS) score, ${ }^{12}$ which was assessed as part of usual care for all patients with stroke in all centres. The mRS score is a common measure of patient functional outcome after stroke, ranging from 0 (no symptoms) to 6 (death). Study staff were instructed to assess mRS scores at 90 days (range 14 days either way). Secondary outcomes were excellent functional outcome (mRS score 0-1), good functional outcome (mRS score 0-2), and favourable functional outcome (mRS score 0-3) at 90 days (range 14 days either way); score on the extended thrombolysis in cerebral infarction scale at the end of the intervention procedure; National Institutes of Health Stroke Scale (NIHSS) score 24-48 hours after intervention; and complications that occurred during intervention, hospital admission, or the three months' follow up period.

We obtained relevant images (baseline noncontrast computed tomography, baseline computed tomography angiography, interventional digital subtraction angiography, and follow-up imaging), which were stored in an imaging database (XNAT; Neuroinformatics Research Group, St Louis, MO) and subsequently analysed by an imaging core laboratory.

\section{Intervention procedure}

Endovascular treatment consisted of arterial catheterisation with a micro-catheter to the level of occlusion, followed by mechanical thrombectomy or thrombus aspiration, or both, with or without delivery of a thrombolytic agent. The method of endovascular treatment for each patient was left to the discretion of the treating doctors.

\section{Study procedures}

Patients were registered through a website (www. mrclean-trial.org/). Registrations were sent by encrypted email to the trial office. The trial office then matched patient data with a specific study identification number. Using this number, all data were entered into a good clinical practice approved, web based clinical database (OpenClinica Community version: 3.12.2). The study coordinators (IGHJ, MJHLM and RBG) checked all centre data for completeness, formatting, and consistency. The study coordinators verified sample source data for all entered data. To check on reporting of safety, we screened discharge letters for complications. For patients transferred from a referring stroke centre to an intervention centre, we collected clinical and imaging data from the referring stroke centre.

\section{Imaging assessment}

Twenty one observers (20 interventional neuroradiologists and one interventional neurologist) at an imaging core laboratory assessed the images. 
The observers were blinded to all clinical findings, with the exception of clinical assessment of the occlusion location in case of baseline non-contrast computed tomography, and were assigned a random subset of images to assess. Before assessment began the observers were provided with guidelines including relevant definitions. In separate sessions, the observers evaluated ASPECTS on baseline noncontrast computed tomography ${ }^{13}$; occluded arterial segment, clot burden score, ${ }^{14}$ and collateral score ${ }^{15}$ on baseline computed tomography angiography; extended thrombolysis in cerebral infarction on digital subtraction angiography ${ }^{16}$; and presence of intracranial haemorrhage on follow up non-contrast computed tomography. ${ }^{17}$

ASPECTS is graded from 0 to 10 , with 1 point subtracted from 10 for any evidence of early ischaemic changes in each defined region on non-contrast computed tomography. ${ }^{13}$ We graded collaterals on a 4 point scale, with 0 for absent collaterals ( $0 \%$ filling of the vascular territory downstream of the occlusion), 1 for poor collaterals ( $>0 \%$ and $\leq 50 \%$ filling of the vascular territory downstream of the occlusion), 2 for moderate collaterals $(>50 \%$ and $<100 \%$ filling of the vascular territory downstream of the occlusion), and 3 for excellent collaterals ( $100 \%$ filling of the vascular territory downstream of the occlusion). ${ }^{15}$ The extended thrombolysis in cerebral infarction score ranges from 0 (no antegrade reperfusion of the occluded vascular territory) to 3 (complete antegrade reperfusion of the occluded vascular territory). ${ }^{16}$ The score includes grade $2 \mathrm{C}$ (slow flow in a few distal cortical vessels or presence of small distal cortical embolisms, corresponding to $90-99 \%$ reperfusion). To reach a score of 2B or higher, it was mandatory to complete digital subtraction angiography runs including anteroposterior and lateral views after endovascular treatment. If a lateral view was missing, 2A was the highest possible score. We classified intracranial haemorrhage on follow-up imaging according to the Heidelberg criteria. ${ }^{17}$

\section{Safety measures}

The complication committee, consisting of two vascular neurologists and one neuroradiologist, evaluated the safety variables on the basis of discharge letters and reports from the imaging core laboratory. To prevent underreporting, one independent investigator (RBG) searched the medical records for complications. The safety variables included mortality within 7,30 , and 90 days of endovascular treatment, symptomatic intracranial haemorrhage, hemicraniectomy, progression of ischaemic stroke (resulting in a decline of at least 4 points on the NIHSS), pneumonia, other infections, cardiac ischaemia, extracranial haemorrhage, and allergic reactions.

We deemed intracranial haemorrhage to be symptomatic if patients died or deteriorated neurologically (a decline of at least 4 points on the NIHSS) and the haemorrhage was related to the clinical deterioration (according to the Heidelberg criteria). To minimise biased reporting, the imaging core laboratory analysed the follow-up images of patients with symptomatic intracranial haemorrhage, and the complication committee made the final decision for reporting a symptomatic intracranial haemorrhage. The complication committee evaluated the progression of ischaemic stroke and new ischaemic stroke on the basis of medical reports of admission.

\section{Missing data}

We retrospectively scored missing NIHSS scores using a standard score chart based on the reported information on neurological examination. If successful reperfusion was not achieved during endovascular treatment, we used the time of last contrast bolus injection as a proxy. Any mRS score of 0 to 5 assessed within 30 days of symptom onset was considered invalid and treated as missing. These values were therefore replaced by mRS scores derived from multiple imputation for the purpose of unbiased estimation of associations of outcome with baseline characteristics and comparisons with MR CLEAN results. ${ }^{18}$ All baseline data and outcomes that are reported are crude and not imputed. We performed multiple imputation with Stata/SE 14.2 (StataCorp, TX) using the variables: age, baseline NIHSS score, glucose level, diabetes, previous myocardial infarction, previous stroke, hypercholesterolemia, atrial fibrillation, drug use (antiplatelet, statin, coumarine, novel oral anticoagulant, and antihypertensive), prestroke mRS score, blood pressure, baseline ASPECTS, occlusion segment, collateral status, time from symptom onset to start of endovascular treatment, time from symptom onset to successful reperfusion or last contrast bolus, extended thrombolysis in cerebral infarction score at the end of the intervention, and NIHSS score after 2448 hours.

\section{Study organisation}

The intervention centres participating in the MR CLEAN trial enrolled patients who received endovascular treatment in the MR CLEAN Registry from the start, after closure of the MR CLEAN trial, and onwards. Local principal investigators were always a senior vascular neurologist and an interventionist. The local principal investigators together formed the steering committee, which met annually and was the final authority on changes to protocol.

\section{The MR CLEAN trial}

The MR CLEAN trial was a multicentre clinical trial with randomised treatment group assignments, which ran from December 2010 to March 2014. This trial compared endovascular treatment plus usual care with usual care alone (control group) in patients with acute ischaemic stroke and a proximal intracranial vessel occlusion in the anterior circulation that was confirmed on imaging. ${ }^{2} 19$

\section{Statistical analysis}

Baseline characteristics are described using standard statistics. We used regression models to compare 
baseline characteristics and outcomes in patients in the MR CLEAN Registry with those in the MR CLEAN intervention and control arms. The effect of endovascular treatment on the mRS score at 90 days in patients in the MR CLEAN Registry compared with the MR CLEAN trial was expressed as an adjusted common odds ratio, derived from multivariable ordinal logistic regression (shift analysis). Because there is consensus on the ordering of the outcome scale in this case (each score on the mRS is more favourable than a 1 point lower score), the common odds ratio can be presented and interpreted as a summary estimate of the treatment effect, even if the underlying proportional odds assumption would be violated. ${ }^{20}$ Therefore, we decided not to formally test this assumption. For all outcome regression analyses using the mRS score, we used the score after multiple imputation. We adjusted for age, sex, baseline NIHSS score, prestroke mRS score, and collateral status. Statistical analyses were performed with Stata/SE 14.2 (StataCorp, TX).

\section{Patient involvement}

No patients were involved in setting the research question or the outcome measures, nor were they involved in developing plans for recruitment, design, or implementation of the study. No patients were asked to advise on interpretation or writing up of results. There are no plans to disseminate the results of the research to study participants or the relevant patient community.

\section{Results}

Overall, 1628 patients were registered in the MR CLEAN Registry between 16 March 2014 and 15 June 2016. For the current analysis, we excluded 140 patients, mostly because of occlusion in the posterior circulation or treatment starting after 6.5 hours from the onset of symptoms (see supplementary figure S1). Therefore 1488 patients were available for final analysis.

\section{Participant characteristics}

Overall, data on 26259 of 26784 of the 18 clinical and radiological baseline characteristics were complete $(98.0 \%)$. The median age of patients was 71 years (interquartile range $60-80$ years), and the median baseline NIHSS score was 16 (interquartile range 1120; table 1). Intravenous alteplase was administered to 1161 patients $(78.0 \%)$, and 808 patients (54.3\%) were transferred from a referring stroke centre to an intervention centre. The median time from onset of symptoms to start of intravenous alteplase treatment was 80 minutes (interquartile range 62120 minutes); from onset of symptoms to start of endovascular treatment was 208 (interquartile range 160-265) minutes; and from onset of symptoms to successful reperfusion or last contrast bolus was 267 (interquartile range 217-331) minutes. Most patients had occlusion of a middle cerebral artery (M1) ( $n=825$; $58.0 \%$ ), followed by an internal carotid artery terminus occlusion (ICA-T) $(\mathrm{n}=313 ; 22.0 \%)$, and M2 occlusion $(\mathrm{n}=175 ; 12.3 \%)$. Ninety five patients $(6.5 \%)$ had poor ASPECTS (0-4) on baseline non-contrast computed tomography, and 565 patients (40.9\%) had absent or poor collaterals on baseline computed tomography angiography.

Major differences in baseline characteristics between patients in the MR CLEAN Registry and those in the MR CLEAN trial intervention arm were age ( 71 v 66 years), intravenous alteplase treatment $(78.0 \% \vee 87.1 \%)$, prestroke mRS score $>2(11.7 \% \vee 4.3 \%)$, transfer from a referring stroke centre $(54.4 \% \mathrm{~V} 44.2 \%)$, onset to start of endovascular treatment (208 v 260 minutes), and onset to successful reperfusion or last contrast bolus (267 v 339 minutes).

\section{Intervention characteristics}

In 80 of the 1488 patients (5.4\%) the target occlusion was not accessible, mostly because of an elongated carotid artery or aortic arch, or an occlusion or stenosis of the carotid artery that could not be passed. In 119 patients $(8.0 \%)$, no target occlusion was present on digital subtraction angiography due to spontaneous reperfusion or distal migration of the clot beyond the M2 or anterior cerebral artery (A2 segment). Intravenous alteplase was administered in 108 out of 119 patients $(90.8 \%)$ and therefore possibly caused migration or dissolvement of the thrombus before endovascular treatment. In nine patients $(0.6 \%)$, the local interventionist ended the procedure before thrombectomy was attempted, despite the presence of a target occlusion. Reasons for this were clinical recovery of the patient $(n=3)$, vessel rupture $(n=1)$, expiration of the six hour time window $(n=1)$, and unknown $(n=4)$. Of the 1280 patients $(86.0 \%)$ in whom thrombus retrieval was attempted, the primary treatment modality was registered in 1220 (95.3\%): stent retriever $(\mathrm{n}=969 ; 79.4 \%)$, aspiration device $(n=207 ; 17.0 \%)$, local delivery of a thrombolytic agent $(n=10 ; 0.8 \%)$, or a different approach $(n=34$; $2.8 \%$ ). A second treatment option was used in 314 of these 1220 (25.7\%) patients and a third in 52 (4.3\%) patients. The median duration of the procedure was 64 minutes (interquartile range 40-90). In 810 of 1488 patients (54.4\%) only local anaesthesia at the groin puncture site was given. General anaesthesia was used in 379 patients $(25.5 \%)$ and conscious sedation in 173 patients $(11.6 \%)$. Data on anaesthetic management were missing for 126 patients $(8.5 \%)$.

\section{Primary outcome}

The mRS score was obtained for all 1488 patients, and multiple imputation was performed in 125 patients $(8.4 \%)$ in whom the mRS score was assessed within 30 days (see supplementary figure S2), because of the lower reliability of this early assessment in a hospital setting. Of the imputed variables, 33111 of the 34224 (96.7\%) were complete. After adjustment for age, sex, baseline NIHSS score, collateral status, and pre-stroke mRS score, the shift towards better functional outcome was significant for patients in the MR CLEAN Registry compared with those in the MR 


\begin{tabular}{|c|c|c|c|}
\hline Characteristics & $\begin{array}{l}\text { MR CLEAN Registry } \\
(n=1488)\end{array}$ & Intervention arm $(n=233)$ & Control arm $(n=267)$ \\
\hline Men & $794(53.3)$ & $135(57.9)$ & $157(58.8)$ \\
\hline Median (interquartile range) NIHSS score & $16(11-20)$ & $17(14-21)$ & $18(14-22)$ \\
\hline Clinical localisation: left hemisphere & $745(53.5)$ & $116(49.8)$ & $153(57.3)$ \\
\hline \multicolumn{4}{|l|}{ Medical history: } \\
\hline Atrial fibrillation & $327(22.3)$ & $66(28.3)$ & $69(25.8)$ \\
\hline Hypertension & $745(50.7)$ & $98(42.1)$ & $129(48.3)$ \\
\hline Diabetes mellitus & $255(17.2)$ & $34(14.6)$ & $34(12.7)$ \\
\hline Myocardial infarction & $228(15.6)$ & $33(14.2)$ & $42(15.7)$ \\
\hline Peripheral artery disease & $135(9.3)$ & $8(3.4)$ & $16(6.0)$ \\
\hline Ischaemic stroke & $249(16.8)$ & $29(12.4)$ & $25(9.4)$ \\
\hline$\geq 3$ & $171(11.7)$ & $10(4.3)$ & $11(4.1)$ \\
\hline Current smoking & $338(22.9)$ & $65(27.9)$ & $78(29.2)$ \\
\hline \multicolumn{4}{|l|}{ Drug use: } \\
\hline Statin & $522(35.8)$ & 65 (27.9) & $78(29.2)$ \\
\hline Antiplatelet & $493(33.6)$ & $64(27.5)$ & $80(30.0)$ \\
\hline \multicolumn{4}{|l|}{ Level of occlusion on non-invasive vessel imaging: } \\
\hline ICA & $82(5.7)$ & $1(0.4)$ & $3(1.1)$ \\
\hline ICA-T & $313(22.0)$ & $59(25.3)$ & $75(28.2)$ \\
\hline M1 & $825(58.0)$ & $154(66.1)$ & $165(62.0)$ \\
\hline M2 & $175(12.3)$ & $18(7.7)$ & $21(7.9)$ \\
\hline Other* & $27(1.9)$ & $1(0.4)$ & $2(0.8)$ \\
\hline \multicolumn{4}{|l|}{ ASPECTS subgroups: } \\
\hline $0-4$ & $93(6.5)$ & $10(4.7)$ & $19(7.2)$ \\
\hline \multicolumn{4}{|l|}{ Categories after onset of stroke (median (interquartile range)): } \\
\hline To intravenous alteplase treatment (mins) & $80(62-120)$ & $85(67-110)$ & $87(65-116)$ \\
\hline To intervention centre (mins) & $134(60-189)$ & $90(48-186)$ & $104(49-209)$ \\
\hline To endovascular treatment (mins) & $208(160-265)$ & $260(210-313)$ & NA \\
\hline Median (interquartile range) to successful reperfusion or last contrast bolus (mins) & $267(217-331)$ & $339(274-395)$ & NA \\
\hline
\end{tabular}

ICA=internal carotid artery; ICA-T=internal carotid artery terminus; M1/M2=middle cerebral artery; NA= not applicable.

*In nine patients the occlusion location was considered to be in the middle cerebral artery (M2) at the moment endovascular treatment was decided, but the imaging core laboratory observed an M3 occlusion. Six patients had a proximal occlusion in the anterior cerebral artery (A1/A2). Twelve patients underwent endovascular treatment without a definitive occlusion on computed tomography angiography according to the core laboratory.

CLEAN intervention arm (adjusted common odds ratio 1.30, 95\% confidence interval 1.02 to $1.67 ; \mathrm{P}=0.03$ ) and control arm $(1.85,1.64$ to $2.34 ; \mathrm{P}<0.01$; fig 1$)$. The corresponding unadjusted values were 1.05 ( 0.83 to 1.32 ) and 1.49 (1.19 to 1.85 ). These associations were similar when data without imputations were analysed (see supplementary table S3).

\section{Secondary outcomes}

In the MR CLEAN Registry, good functional outcome (mRS score 0-2) was achieved in 517 of 1363 patients (37.9\%; table 2), compared with $32.6 \%$ in the MR CLEAN intervention arm and $19.1 \%$ in the control arm. The NIHSS score at 24-48 hours after endovascular treatment was available in 1274 of the 1400 (91.0\%) patients who were still alive. For these patients, the median NIHSS score was 11 (interquartile range 4-17; table 2 ), and for 236 patients (16.9\%) the NIHSS score was 2 or lower. On average, the NIHSS score improved by 4 points (interquartile range 0-9) between baseline and 24-48 hours after stroke onset. Compared with patients in the MR CLEAN trial intervention and control arms those in the MR CLEAN Registry achieved statistically significantly better scores for all secondary outcomes, with the exception of successful reperfusion (see supplementary table S1).

Since the time from onset of symptoms to start of endovascular treatment was shorter in patients in 


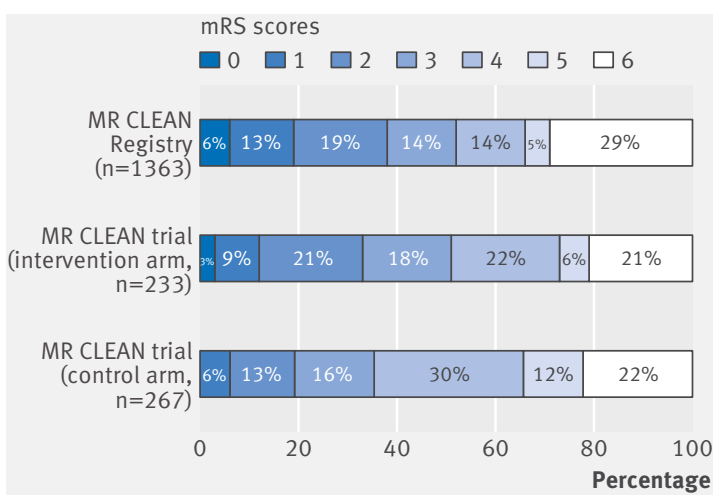

Fig 1 | Distribution of modified Rankin Scale (mRS) score at 90 days in MR CLEAN Registry (before imputation) and MR CLEAN trial intervention and control arms. The proportion of deaths is calculated as 398 of 1363 patients instead of 1488 patients and therefore inflated by $2 \%$ because in this figure the denominator does not include 125 patients with missing mRS scores. The mRS category 0 for the MR CLEAN control arm is small $(n=1$, $0.4 \%$ ) and therefore not visible in the figure

the MR CLEAN Registry compared with those in the MR CLEAN trial intervention arm, we did additional analysis adjusting for this variable. This resulted in a smaller treatment effect and also in the absence of a statistically significant difference in functional outcome between patients in the MR CLEAN Registry and those in the MR CLEAN trial intervention arm (adjusted common odds ratio 1.11, 95\% confidence interval 0.86 to $1.43 ; \mathrm{P}=0.43)$. The two studies also showed clear differences between baseline systolic blood pressure and treatment with intravenous alteplase. Additional adjustments for these two factors increased the effect of endovascular treatment in patients in the MR CLEAN Registry compared with those in the MR CLEAN trial intervention and control arms (see supplementary table S4).

Of all patients analysed, $1467(98.6 \%)$ had an extended thrombolysis in cerebral infarction score available after intervention. Successful reperfusion was achieved in 849 of 1467 patients (57.9\%). In the 1280 cases in which an actual endovascular treatment device was used, reperfusion status (extended thrombolysis in cerebral infarction score) was available for 1266 patients $(98.9 \%)$. In the remaining 14 patients $(1.1 \%)$, intervention images were unavailable or of insufficient quality, and no extended thrombolysis in cerebral infarction score was ascertained. Of these 1266 patients with extended thrombolysis in cerebral infarction scores, 743 (58.7\%) had successful reperfusion (score $2 \mathrm{~B}: \mathrm{n}=239$; score $2 \mathrm{C}$ : $\mathrm{n}=125$; and score 3: $n=379$; table 2). For 199 patients (15.7\%), a lateral digital subtraction angiography projection after treatment was not obtained, and $2 \mathrm{~A}$ was the highest achievable score.

In patients without imputed mRS scores, death within 90 days occurred in 398 of 1363 (29.2\%) in the MR CLEAN Registry compared with $21.0 \%$ and $22.1 \%$ in MR CLEAN intervention and control arms, respectively (table 3 and supplementary table S2). In 86 out of 1488 patients (5.8\%) symptomatic intracranial haemorrhage occurred compared with $7.7 \%$ and $6.4 \%$ in the MR CLEAN intervention and control arms, respectively. The MR CLEAN Registry was comparable to the MR CLEAN trial intervention and control arms for mortality, symptomatic intracranial haemorrhage, and almost all other complications. Only hemicraniectomy and progression of ischaemic stroke occurred less often in patients in the MR CLEAN Registry.

\section{Discussion}

The results of our study show that endovascular treatment in routine clinical practice, for patients recorded in the MR CLEAN Registry with acute ischaemic stroke, due to proximal intracranial vessel occlusion in the anterior circulation, is at least as effective and safe as in the MR CLEAN trial. A higher proportion of patients reached good functional outcome after 90 days, despite older age and more comorbidities. Times from stroke onset to start of endovascular treatment and from onset to successful reperfusion or last contrast bolus improved in patients in the MR CLEAN Registry compared with those in the MR CLEAN trial, and comparable reperfusion rates were achieved.

\section{Limitations of this study}

The MR CLEAN Registry is a large, consecutive, nationwide study, representative of current clinical

\begin{tabular}{|c|c|c|c|}
\hline \multirow[b]{2}{*}{ Scores } & \multirow[b]{2}{*}{ MR CLEAN Registry $(n=1488)^{\star}$} & \multicolumn{2}{|l|}{ MR CLEAN } \\
\hline & & Intervention arm $(n=233)$ & Control arm $(n=267)$ \\
\hline Median (interquartile range) mRS score at 90 days & $3(2-6)$ & $3(2-5)$ & $4(3-5)$ \\
\hline \multicolumn{4}{|l|}{ mRS score at 90 days } \\
\hline $0-1$ & $258(18.9)$ & $27(11.6)$ & $16(6.0)$ \\
\hline $0-2$ & $517(37.9)$ & $76(32.6)$ & $51(19.1)$ \\
\hline $0-3$ & $710(52.1)$ & $119(51.1)$ & $95(35.6)$ \\
\hline Successful reperfusion (eTICI grade $2 \mathrm{~B}-3) \dagger$ & $743 / 1266(58.7)$ & $115 / 196(58.7)$ & NA \\
\hline Median (interquartile range) NIHSS score post-intervention (24 hours) & $11(4-17)$ & $13(6-20)$ & $16(12-21)$ \\
\hline
\end{tabular}




\begin{tabular}{|c|c|c|c|}
\hline Complications & MR CLEAN Registry $(n=1488)$ & \multicolumn{2}{|l|}{ MR CLEAN } \\
\hline \multicolumn{4}{|l|}{ Mortality: } \\
\hline 7 days & $207(15.2)^{\star}$ & $33(14.2)$ & $27(10.1)$ \\
\hline 30 days & $328(24.1)^{\star}$ & $44(18.9)$ & $49(18.4)$ \\
\hline Symptomatic intracranial haemorrhage & $86(5.8)$ & $18(7.7)$ & $17(6.4)$ \\
\hline Hemicraniectomy & $32(2.2)$ & $14(6.0)$ & $13(4.9)$ \\
\hline Progression of ischaemic stroke & $140(9.4)$ & $46(19.7)$ & $47(17.6)$ \\
\hline Pneumonia & $184(12.3)$ & $25(10.7)$ & $41(15.4)$ \\
\hline Other infection & $75(5.0)$ & $16(6.9)$ & $9(3.4)$ \\
\hline
\end{tabular}

practice, and with core laboratory evaluation of all images and complications. However, there were still several limitations. Firstly, inherent to a registry study, non-registration of treated patients might be problematic. As registration of treatment was a prerequisite for reimbursement, we think that underregistration is minimal. Secondly, no central outcome adjudication was available for our study, so we relied on local investigators for reporting the functional outcome at 90 days, possibly introducing bias. Optimistic reports from local investigators could have resulted in a larger proportion of functionally independent patients. However, stroke or research nurses not involved in the treatment of patients primarily performed outcome assessment, and according to standard questionnaires. Thirdly, not all digital subtraction angiography images were of sufficient quality for optimal assessment of successful reperfusion, because sometimes full anteroposterior or lateral runs were not available. This probably resulted in underreporting of successful reperfusion, as a maximum score of extended thrombolysis in cerebral infarction 2A could be achieved in those cases. Fourthly, we report data from the Netherlands only. Extrapolation of our results to other countries should be done with caution, taking into account differences in logistics, experience and technical expertise of interventionists, local guidelines, and availability of resources. Our broad entry criteria could, however, enhance the generalisability. Finally, patients with ischaemic stroke due to occlusion of an intracranial proximal artery were not registered in the database if they did not undergo endovascular treatment. Consequently, we are unaware of the reason why endovascular treatment was not performed in these patients. Patient selection bias based on a changed clinical status, or patient characteristics such as comorbidity and being of an older age group (>80 years old), could have occurred. However, this is one of the few studies in which a relatively large number of patients with absent or poor collaterals were treated, which emphasises that patients with poor prognostic factors were not systematically excluded from endovascular treatment.
Since the widespread implementation of endovascular treatment as standard of care in the Netherlands, two studies describing outcomes of endovascular treatment in routine clinical practice have been published. The first included 77 patients with ischaemic stroke in the anterior circulation. ${ }^{21}$ The second was a multicentre registry, which prospectively enrolled 984 patients with any confirmed large vessel occlusion (including in the posterior circulation), without other prespecified imaging selection. ${ }^{22}$ Consecutive inclusion was not required. Treatment had to be started within eight hours after the onset of symptoms. Good functional outcome (mRS score 0 -2) was achieved in $56.5 \%$, successful reperfusion (extended thrombolysis in cerebral infarction score 2B-3) in $87.9 \%$, and symptomatic intracranial haemorrhage within 24 hours in $1.4 \%$ of patients. However, in contrast with the MR CLEAN Registry, no patients with prestroke functional dependency were enrolled, patients were slightly younger, and inclusion was restricted to patients with baseline NIHSS scores of 8 or more or 30 or less, which might reduce the degree to which these results represent current routine clinical practice. Moreover, patients had to be treated with a thrombectomy device (Solitaire or Mindframe) from one company, whereas in the MR CLEAN Registry this was left to discretion of the interventionist.

Despite consistent proportions of patients who experienced successful reperfusion in the MR CLEAN Registry and the MR CLEAN trial, the improved functional outcome in patients in the MR CLEAN Registry can be explained by a shorter time from onset of symptoms to successful reperfusion or last contrast bolus of more than one hour. After additional adjustment for this variable, the difference in benefit of endovascular treatment between the MR CLEAN Registry and the MR CLEAN trial intervention arm decreased and was no longer statistically significant. This again emphasises the importance of decreasing time from symptom onset to start of endovascular treatment as well as procedure times. Faster successful reperfusion might also have contributed to the lower rates of hemicraniectomy and stroke progression 
in patients in the MR CLEAN Registry. Increased experience of the interventionists could also have contributed to the rate of good functional outcome in routine clinical practice. Finally, the proportion of patients who died within 90 days of onset of symptoms was higher than those in the MR CLEAN trial. This could be explained by a broader population being considered for endovascular treatment in routine clinical practice, including patients of greater age and prestroke morbidity.

The results of our study might have important implications for the future of endovascular treatment for acute ischaemic stroke. We found that in routine clinical practice, endovascular treatment started within 6.5 hours after stroke onset shows results comparable to the trial setting (we chose to extend the time window by a half hour compared with that in the MR CLEAN trial, to be compatible with the trial inclusion criterion of intended treatment within six hours). These outcomes are reported in a broad group of patients without imaging selection (apart from computed tomography angiography to confirm occlusion of proximal vessels), in contrast with the trend in current clinical trials to select patients before treatment with a favourable prognosis based on imaging characteristics. However, some patient selection probably also occurred in this registry, because treatment decisions were made based on individual considerations by doctors.

The MR CLEAN Registry elucidates that, despite much research over the past years, patient selection for endovascular treatment is still not applied in a structured manner in clinical practice, at least in the Netherlands. Despite this, patients' functional outcome improved substantially compared with the MR CLEAN trial. Research is needed to improve existing patient selection paradigms and tools, particularly those combining clinical and imaging variables, ${ }^{23}$ and to provide consensus on how patient selection for endovascular treatment can be reliably and consistently implemented.

\section{Conclusion}

In routine clinical practice, endovascular treatment for patients with acute ischaemic stroke, due to proximal intracranial vessel occlusion in the anterior circulation, is at least as effective and safe as in the setting of a randomised controlled trial.

Ivo G H Jansen, medical researcher, Maxim J H L Mulder, medical researcher, Robert-Jan B Goldhoorn, medical researcher, Bart J Emmer, interventional radiologist, Adriaan C G M van Es, interventional radiologist, Bob Roozenbeek, neurologist, Wouter J Schonewille, neurologist, René van den Berg, interventional radiologist, Jonathan M Coutinho, neurologist, Julie Staals, neurologist, Alida A Postma, interventional radiologist, Geert J Lycklama à Nijeholt, interventional radiologist, Jeannette Hofmeijer, neurologist, Jasper M Martens, interventional radiologist, Heleen M den Hertog, neurologist, Emiel J C Sturm, interventional radiologist, $\mathrm{H}$ Bart van der Worp, neurologist, Rob H Lo, interventional radiologist, Roel J J Heijboer, interventional radiologist, Koos Keizer, neurologist, Maarten Uyttenboogaart, interventional neurologist, Omid Eshghi, interventional radiologist, Marieke J H Wermer, neurologist, Marianne A A van Walderveen, interventional radiologist, Ewoud J van Dijk, neurologist, Hieronymus D Boogaarts, interventional radiologist, Sebastiaan F de Bruijn, neurologist, Lukas C van Dijk, interventional radiologist, Jan S P van den Berg, neurologist, Boudewijn A A M van Hasselt, interventional radiologist, Paul L M de Kort, neurologist, Jo P P Peluso, interventional radiologist, Tobien H C M L Schreuder, neurologist, Leo A M Aerden, neurologist, René J Dallinga, interventional radiologist, Marieke E S Sprengers, interventional radiologist, Lonneke S F Yo, interventional radiologist, Sjoerd $F M$ Jenniskens, interventional radiologist, Stefan D Roosendaal, interventional radiologist, Bas F W van der Kallen, interventional radiologist, Ido R van den Wijngaard, interventional neurologist, Joost C J Bot, interventional radiologist, Pieter J van Doormaal, interventional radiologist, $\mathrm{H}$ Zwenneke Flach, interventional radiologist, Esmee Venema, medical researcher, Hester F Lingsma, senior medical statistician, Ludo F M Beenen, radiologist, Albert J Yoo, interventional radiologist, Jelis Boiten, neurologist, Jan A Vos, interventional radiologist, Yvo B W E M Roos, professor of acute neurology, Robert I van Oostenbrugge, professor of vascular neurology, Aad van der Lugt, professor of neuroradiology and head/ neck radiology, Wim $\mathrm{H}$ van Zwam, interventional radiologist, Diederik W J Dippel, professor of neurovascular diseases, Charles B L M Majoie professor of neuroradiology, for the MR CLEAN Registry investigators (see supplementary file for full details of authors' affiliations).

Executive committee: DWJD, AvdL, CBLMM, YBWEMR, RJvO, WHvZ, $J B$, and JAV; study coordinators: IGHJ, MJHLM, and RBG; local principal investigators: WJS, JAV, CBLMM, JMC, MJHW, MAAvW, JS, WHVZ, JH, JMM, GJLàN, JB, BR BJE, SFdB, LCvD, HBvdW, RHL, EJvD, HDB, PLMdK JJPP, JSPvdB, BAAMvH, LAMA, RJD, MU, OE, THCMLS, RJJH, KK, LSFY, $\mathrm{HMdH}$, and EJCS; imaging assessment committee: CBLMM (chair), WHvZ, AvdL, GJLàN, MAAvW, MESS, SFMJ, RvdB, AJY, LFMB, SDR, BFWvdK, IRvdW, AvE, BE, JMM, LSFY, JAV, JCJB, and P-JvD; writing committee: DWJD (chair), AvdL, CBLMM, YBWEMR, RJvO, WHvZ, GJLàN, JB, JAV, WJS, JH, JMM, HBvdW, and RHL; adverse event committee: RJvO (chair), JH, and HZF; trial methodologist: HFL

Research nurses/local trial coordinators, based in Departments of Neurology: Naziha el Ghannouti and Martin Sterrenberg, (Erasmus MC University Medical Centre, Rotterdam, Netherlands); Corina Puppels and Wilma Pellikaan (Sint Antonius Hospital, Nieuwegein, Netherlands); Rita Sprengers (Department of Radiology, Academic Medical Centre, Amsterdam, Netherlands); Marjan Elfrink (Rijnstate Hospital, Arnhem, Netherlands); Joke de Meris and Tamara Vermeulen (Haaglanden MC, The Hague, Netherlands); Annet Geerlings (Radboud University Medical Centre, Nijmegen, Netherlands), Gina van Vemde (Isala Klinieken, Zwolle, Netherlands); Tiny Simons (Atrium Medical Centre, Heerlen, Netherlands); Cathelijn van Rijswijk (Sint Elisabeth Hospital, Tilburg, Netherlands); Gert Messchendorp (University Medical Centre Groningen, Groningen, Netherlands); Hester Bongenaar (Catharina Hospital, Eindhoven, Netherlands); Karin Bodde (Reinier de Graaf Gasthuis, Delft, Netherlands); Sandra Kleijn, Jasmijn Lodico, and Hanneke Droste (Medical Spectrum Twente, Enschede, Netherlands); Maureen Wollaert and Daisy Ramakers (Maastricht University Medical Centre and Cardiovascular Research Institute Maastricht (CARIM), Maastricht, Netherlands); Erna Bos (Leiden University Medical Centre, Leiden, Netherlands); Yvonne Drabbe, and Mostafa Khalilzada (HAGA Hospital, The Hague, Netherlands); and Berber Zweedijk (University Medical Centre Utrecht, Utrecht, Netherlands).

PhD/Medical students: Vicky Chalos (Department of Neurology, Erasmus MC University Medical Centre, and Public Health, Erasmus MC University Medical Centre, Rotterdam, Netherlands); Kars I Compagne (Department of Radiology, Erasmus MC University Medical Centre, Rotterdam, Netherlands); Ralph R Geuskens (Department of Radiology, Academic Medical Centre, Amsterdam, Netherlands); Tim van Straaten (Department of Neurology, Radboud University Medical Centre, Nijmegen, Netherlands); Saliha Ergezen, Roger RM Harmsma, Daan Muijres, and Anouk de Jong (Department of Neurology, Erasmus MC University Medical Centre, Rotterdam, Netherlands); Wouter Hinseveld (Department of Neurology, Sint Antonius Hospital, Nieuwegein, Netherlands); Olvert A Berkhemer (Department of Neurology, Erasmus MC University Medical Centre, Rotterdam, Netherlands, and Department of Radiology, Academic Medical Centre, Amsterdam, Netherlands); Anna MM Boers (Department of Radiology, and Department of Biomedical Engineering and Physics, Academic Medical Centre, Amsterdam, Netherlands); J Huguet, Paul FC Groot, Marieke A Mens, Katinka R van Kranendonk, Kilian M Treurniet, Manon Kappelhof, Manon L Tolhuijsen, and Heitor Alves (Department of Radiology, Academic Medical Centre, Amsterdam, Netherlands).

Contributors: IGHJ, MJHLM, and RBG prepared the first draft. MJHLM, EV, HFL, and DWJD designed and did the statistical analyses. IGHJ, MJHLM, RBG, DWJD, CBLMM, RJVO, JAV, JB, YBWEMR, AvdL, and WHVZ participated in study design, data collection, data analysis, interpretation, and writing of the manuscript. GJLàN, MAAvW, MESS, SFMJ, RvdB, AJY, LFMB, SDR, BFWvdK, IRvdW, AvE, BJE, JMM, LSFY, 
$J \mathrm{~B}$, and PJVD participated in data analysis. IGHJ is the guarantor. The study coordinators, local investigators, and members of the executive, imaging, and complication committees designed the study, collected and analysed the data, wrote the manuscript, and made the decision to submit the manuscript for publication. The authors vouch for the accuracy and completeness of the data and for the fidelity of this report to the study protocol. All authors participated in patient enrolment and data collection, critically reviewed the manuscript, and approved the final version. IGHJ, MJHLM and RBG contributed equally, as did JB, JAV, YBWEMR, RJvO, AvdL, WHVZ, DWJD, and CBLMM.

Funding: This study was funded and carried out by the Erasmus University Medical Centre, the Academic Medical Centre Amsterdam, and the Maastricht University Medical Centre. The study was additionally funded by the Applied Scientific Institute for Neuromodulation (Toegepast Wetenschappelijk Instituut voor Neuromodulatie), which played no role in trial design and patient enrolment, nor in data collection, analysis, or writing of the manuscript. The corresponding author had full access to all the data. The executive committee had responsibility for the decision to submit for publication.

Competing interests: All authors have completed the ICMJE uniform disclosure form at www.icmje.org/coi_disclosure.pdf and declare: BJE reports grants from Stryker Neurovascular and personal fees from Dekra and from Novartis, outside the submitted work. CBLMM reports grants from TWIN, during the conduct of the study and grants from CVON/Dutch Heart Foundation and from Stryker outside the submitted work (paid to institution). AAP reports personal fees from Bayer and from Siemens outside the submitted work. WHVZ reports personal fees from Codman and from Stryker outside the submitted work. RvdB reports a consultancy agreement with Codman Depuy outside the submitted work. HBW reports grants from the Dutch Heart Foundation outside the submitted work. AJY reports grants from Penumbra and Cerenovus/Neuravi and equity ownership interest from Insera Therapeutics outside the submitted work. AvdL reports consulting fees from Stryker outside the submitted work. DWJD reports grants from the Dutch Heart Foundation, AngioCare, Medtronic/Covidien/ EV3, MEDAC/LAMEPRO, Penumbra, Top Medical/Concentric, and Stryker during conduct of the study; consultation fees from Stryker, Bracco Imaging, and Servier, received by the Erasmus University Medical Centre, outside the submitted work. All other authors did not receive support from any organisation for the submitted work, had no financial relationships with any organisations that might have an interest in the submitted work in the previous three years, and had no other relationships or activities that could appear to have influenced the submitted work.

Ethical approval: The central medical ethics committee of the Erasmus Medical Centre Rotterdam, the Netherlands, evaluated the study protocol (see supplementary file) and granted permission to carry out the study as a registry.

Data sharing: No additional data available.

Transparency: The lead author (IGHJ) affirms that this manuscript is an honest, accurate, and transparent account of the study being reported; that no important aspects of the study have been omitted; and that any discrepancies from the study as planned (and, if relevant, registered) have been explained.

This is an Open Access article distributed in accordance with the Creative Commons Attribution Non Commercial (CC BY-NC 4.0) license, which permits others to distribute, remix, adapt, build upon this work non-commercially, and license their derivative works on different terms, provided the original work is properly cited and the use is noncommercial. See: http://creativecommons.org/licenses/by-nc/4.0/.

1 Badhiwala JH, Nassiri F, Alhazzani W, et al. Endovascular Thrombectomy for Acute Ischemic Stroke: A Meta-analysis. JAMA 2015;314:1832-43. 10.1001/jama.2015.13767

2 Berkhemer OA, Fransen PS, Beumer D, et al, MR CLEAN Investigators. A randomized trial of intraarterial treatment for acute ischemic stroke. N Engl J Med 2015;372:11-20. 10.1056/NEJMoa1411587

3 Campbell BC, Mitchell PJ, Kleinig TJ, et al, EXTEND-IA Investigators. Endovascular therapy for ischemic stroke with perfusionimaging selection. N Engl J Med 2015;372:1009-18. 10.1056/ NEJMoa1414792

4 Goyal M, Demchuk AM, Menon BK, et al, ESCAPE Trial Investigators. Randomized assessment of rapid endovascular treatment of ischemic stroke. N Engl J Med 2015;372:1019-30. 10.1056/ NEJMoa1414905

5 Jovin TG, Chamorro A, Cobo E, et al, REVASCAT Trial Investigators. Thrombectomy within 8 hours after symptom onset in ischemic stroke. N Engl J Med 2015;372:2296-306. 10.1056/ NEIMoa1503780
6 Saver JL, Goyal M, Bonafe A, et al, SWIFT PRIME Investigators. Stentretriever thrombectomy after intravenous t-PA vs. t-PA alone in stroke. N Engl I Med 2015;372:2285-95. 10.1056/NEJMoa1415061

7 Bracard S, Ducrocq X, Mas JL, et al, THRACE investigators. Mechanical thrombectomy after intravenous alteplase versus alteplase alone after stroke (THRACE): a randomised controlled trial. Lancet Neurol 2016;15:1138-47. 10.1016/S1474-4422(16)30177-6

8 Muir KW, Ford GA, Messow CM, et al, PISTE Investigators. Endovascular therapy for acute ischaemic stroke: the Pragmatic Ischaemic Stroke Thrombectomy Evaluation (PISTE) randomised, controlled trial. J Neurol Neurosurg Psychiatry 2017;88:38-44. 10.1136/jnnp-2016-314117

9 Goyal M, Menon BK, van Zwam WH, et al, HERMES collaborators. Endovascular thrombectomy after large-vessel ischaemic stroke: a meta-analysis of individual patient data from five randomised trials. Lancet 2016;387:1723-31. 10.1016/S01406736(16)00163-X

10 Rothwell PM. External validity of randomised controlled trials: "to whom do the results of this trial apply?" Lancet 2005;365:82-93. 10.1016/S0140-6736(04)17670-8

11 Frieden TR. Evidence for Health Decision Making - Beyond Randomized, Controlled Trials. N Engl J Med 2017;377:465-75 10.1056/NEIMra1614394

12 van Swieten JC, Koudstaal PJ, Visser MC, Schouten HJ, van Gijn J. Interobserver agreement for the assessment of handicap in stroke patients. Stroke 1988;19:604-7. 10.1161/01.STR.19.5.604

13 Barber PA, Demchuk AM, Zhang J, Buchan AM. Validity and reliability of a quantitative computed tomography score in predicting outcome of hyperacute stroke before thrombolytic therapy. ASPECTS Study Group. Alberta Stroke Programme Early CT Score. Lancet 2000;355:1670-4. 10.1016/S0140-6736(00)02237-6

14 Puetz V, Dzialowski I, Hill MD, et al, Calgary CTA Study Group. Intracranial thrombus extent predicts clinical outcome, final infarct size and hemorrhagic transformation in ischemic stroke: the clot burden score. Int J Stroke 2008;3:230-6. 10.1111/j.17474949.2008.00221.x

15 Tan IY, Demchuk AM, Hopyan J, et al. CT angiography clot burden score and collateral score: correlation with clinical and radiologic outcomes in acute middle cerebral artery infarct. AJNR Am J Neuroradiol 2009;30:525-31. 10.3174/ajnr.A1408

16 Noser EA, Shaltoni HM, Hall CE, et al. Aggressive mechanical clot disruption: a safe adjunct to thrombolytic therapy in acute stroke? Stroke 2005·36:292-6.10.1161/01.STR 0000152331.93770.18

17 von Kummer R, Broderick JP, Campbell BC, et al. The Heidelberg Bleeding Classification: Classification of Bleeding Events After Ischemic Stroke and Reperfusion Therapy. Stroke 2015;46:2981-6. 10.1161/STROKEAHA.115.010049

18 Donders AR, van der Heijden GJ, Stijnen T, Moons KG. Review: a gentle introduction to imputation of missing values. J Clin Epidemiol 2006;59:1087-91. 10.1016/j.jclinepi.2006.01.014

19 Fransen PS, Beumer D, Berkhemer OA, et al, MR CLEAN Investigators. MR CLEAN, a multicenter randomized clinical trial of endovascular reatment for acute ischemic stroke in the Netherlands: study protocol for a randomized controlled trial. Trials 2014;15:343. 10.1186/1745-6215-15-343

20 Senn S, Julious S. Measurement in clinical trials: a neglected issue for statisticians? Stat Med 2009;28:3189-209. 10.1002/sim.3603

21 Carvalho A, Cunha A, Rodrigues M, et al. Mechanical Thrombectomy in Acute Ischemic Stroke: Initial Single-Center Experience and Comparison with Randomized Controlled Trials. J Stroke Cerebrovasc Dis 2017;26:589-94. 10.1016/j. istrokecerebrovasdis.2016.11.116

22 Mueller-Kronast NH, Zaidat OO, Froehler MT, et al, STRATIS Investigators. Systematic Evaluation of Patients Treated With Neurothrombectomy Devices for Acute Ischemic Stroke: Primary Results of the STRATIS Registry. Stroke 2017;48:2760-8. 10.1161/ STROKEAHA.117.016456

23 Venema E, Mulder MJHL, Roozenbeek B, et al. Selection of patients for intra-arterial treatment for acute ischaemic stroke: development and validation of a clinical decision tool in two randomised trials. BMJ 2017;357:j1710. 10.1136/bmj.j1710

Supplementary information: Tables S1-4; fig S1, flowchart of MR CLEAN Registry patients selected for core analysis; and fig S2, modified Rankin scale (mRS) score before multiple imputation compared to the $\mathrm{mRS}$ score after multiple imputation. Any mRS score of 0 to 5 assessed within 30 days was treated as missing and replaced by multiple imputation

Supplementary information: Authors' affiliations 